\title{
Experimental Foundations of Chemical Engineering
}

R. R. WHITE and S. W. CHURCHILL

University of Michigan, Ann Arbor, Michigan

Fundamental knowledge consists of understanding not only the models which correlate and rationalized observations but also the quantity and quality of the observations. The degree of correlation attempted should be consistent with this quantity and quality.

The scientist correlates and rationalizes observations primarily to broaden his general understanding of the physical universe, and his efforts at correlation are more often than not a way of speculating on the future experiments which should be undertaken to test his concepts. For this purpose he is often satisfied with accuracy to one order of magnitude. The engineer, faced with the design and operation of expensive equipment, is almost always interested in a much higher accuracy in his correlations.

Virtually all existing data in the rate processes are forced into rationalizations based upon either potential-difference or potential-gradient models. The application of these models to systems of even relatively simple geometry gives rise to mathematical complexity beyond present analytic ability. Even so, our ability to measure and observe seems to be far below our ability to analyze. These two models have been used effectively in chemical engineering in the past and have served to organize a great deal of information in the rate processes. Present research efforts appear to be directed primarily toward filling in gaps in available data and in refining the application of the models. We seem to suffer from a lack of models to test and a lack of data designed to test critically the existing models. There is relatively little exploratory research in chemical engineering as compared, for example, to physics.

When one views chemical engineering as a chain extending from economics, markets, mechanical design, process design, and process development to process research, the uncertainties inherent in some of the links easily involve variances of 50 to $80 \%$. Under these circumstances it would appear that the present orientation of research is distributed much too heavily toward refinement of existing ideas and not nearly enough toward exploration.

The observations made by scientists and engineers form the basis of chemical engineering. These observations are collected in a concise form as correlations and rationalized in terms of models or mechanisms, which are referred to as hypotheses, theories, and laws.

It is natural that the consideration of engineering problems is focused on theories. In today's explosive production of engineering data the pressure on the engineer to follow the patterns of thought and technique suggested by existing theory is almost irresistible; thus it is ironic that in a time of "plenty," with respect to engineering accomplishment, the phenomenological bases of chemical engineering seem to be increasingly ignored.

Typical of this tendency is the fact that publication of original data has largely been abandoned in favor of deposition in such repositories as the American Documentation Institute or in the original research records, which are relatively inaccessible. As a result present knowledge, as represented by the literature, has the appearance of correlation resting upon correlation, theory upon theory, and the data presented consist of a few graphs greatly reduced in scale, with only a few major coordinate lines. It is becoming more and more usual to accept the general statements and conclusions of the authors because of the time and trouble involved in obtaining the data necessary to test alternate correlations or even to appraise the quantity or quality of the expreimental work.

While the editors of technical publications justify the omission of experimental data on the grounds of cost, it is the profession that must be blamed. Obviously the profession as a whole values the literature more as a record of theory than as a repository of data.

The rationalization of data in terms of models and mechanisms is of paramount importance in scientific and technological development. However in the absence of an understanding of the quantity and quality of the data being rationalized, rationalization becomes meaningless speculation. The Greeks of ancient times were superb philosophers but poor scientists and engineers because they did not wish to be bothered with experimental measurements. As Alfred North Whitehead stated, "There are these hard and irreducible things we call facts; you hear them, you smell them, you see them, you taste them and you feel them, directly or by instrumental extension. Without them you may think indefinitely and have nothing." An understanding of the phenomenological bases of the rate processes is overwhelmingly important in developing the judgment that is crucial in engineering. It seems fitting, therefore, that this paper, as part of a symposium on fundamentals discusses in a general way the kinds of observations with which chemical engineers are concerned, the implications of the rate concepts which are used to summarize these observations, and the requirements and experience which are peculiar to chemical engineering.

\section{DESCRIPTION OF RATE PROCESSES}

There are two principal aspects to the rate processes, the phenomenological and the theoretical. The phenomenological aspect is concerned primarily with identification of the phenomenon under consideration. The need for identification does not arise from thin air but from highly practical considerations. We wish to rationalize the performance of heat or mass exchangers, or we wish to say 
something about time and the composition of a reacting system, etc. Therefore, we attempt to define processes in such a way that the definition will be useful either for comparing processes under different conditions or for designing equipment in which processes are carried out. Isaac Newton encountered these same problems with respect to velocity and solved them by devising the calculus. The general definition of rate processes

$$
r=\frac{1}{L} \frac{d S}{d \theta}
$$

Where $r$ is the change in some quantity $S$ per unit time $\theta$ per unit extent of the system $L$, is essentially the expression devised by Newton. When applied to heat transfer, $r$ is the heat flux density expressed in British thermal units per hour per square foot, $S$ is energy in British thermal units, and $L$ is the area through which the energy is transferred; when applied to mass transfer, $S$ is the mass of some component transferred per unit time through the area $L$. For a homogeneous chemical reaction $S$ is the mass of some species produced per unit time per unit volume $L$, etc. The sign of the derivative is usually chosen to yield a positive rate.

The rate of a steady state process can alternatively be written as

$$
r=\frac{d s}{d L}
$$

where $s$ is the flux of some quantity and $L$ is again the extent of the system. The rate in each case has the same dimensions as in the corresponding unsteady state process. For heat transfer $s$ is the heat flux in British thermal units per hour, and $L$ is again the area through which the the heat is transferred. For mass transfer $s$ is the component flux in mass per unit time and $L$ is the area through which the component is transferred. For a chemical reaction $s$ is the mass flux of some species and $L$ is the volume of the system.

All these particular definitions incor- porate a time rate of change of a property or quantity per unit extent of the system and, explicitly or implicity, an indication of the direction of the change.

\section{DETERMINATION OF THE RATE}

A definition is essentially a set of instructions as to what to measure in order to determine a rate. Thus in transient heat transfer one must measure the enthalpy or energy of a given system and time or its equivalent. In mass

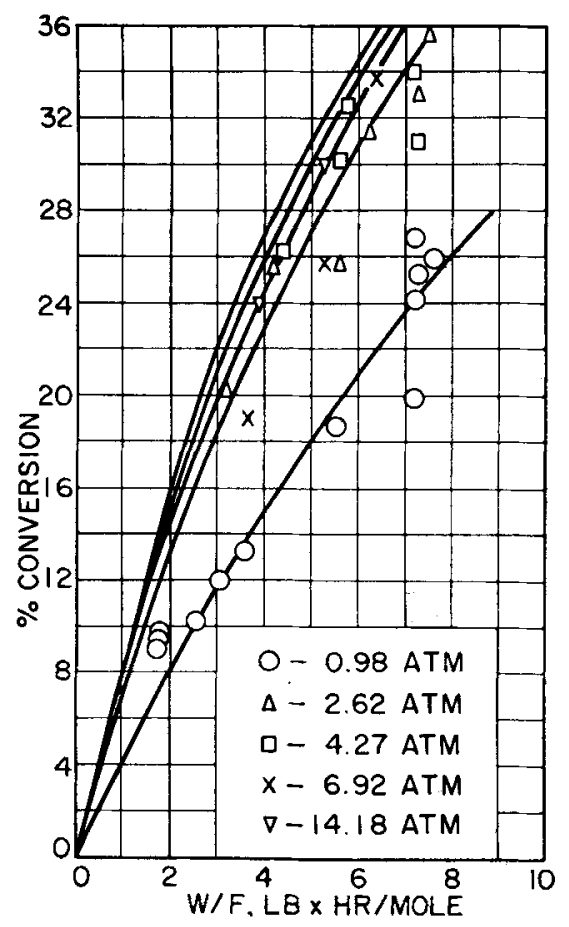

Fig. 1. Conversion as a function of space time.

transfer and chemical reactions the mass of a particular species in a chosen system must be measured as a function of time. In steady state heat transfer the heat flux must be measured as a function of area, etc.

While the basic concepts of the calculus with respect to the limiting process represented by the derivative is taught to, and apparently understood by, most engineering students when presented in the frame of reference of mathematics, it appears to be rather easy to forget these concepts in an engineering frame of reference. One is likely to forget that rate is a concept and that instantaneous or point rates of any kind are very difficult if not impossible to measure directly. Ordinarily one can obtain data from which average rates can be computed over finite intervals of time or over finite areas or volumes, but one is then forced to smoothing or some other interpretative process in order to deduce or estimate instantaneous or point rates. Basically the problem is one of numerical differentiation or smoothing of integral data. Whenever integral data are numerically differentiated, errors or irreproducibilities of the integral data are exaggerated by approximately one order of magnitude. Thus integral data which are precise to $10 \%$ commonly yield values of average rates which are precise only to 50 to $90 \%$.

\section{PROCESS CALCULATIONS}

The use of measured rates in process design involves precisely the same mental operations as the definitions or measurements, since the process calculation is in a sense a hypothetical or mental experiment. This is illustrated by rewriting the definition in the form of Equation (3), which indicates how one would calculate the size of batch equipment required to transport a certain amount of heat or mass in a certain time.

$$
A=\frac{\int-\frac{d S}{r}}{\int d \theta}
$$

A similar integral

$$
A=\int \frac{d s}{r}
$$

arises for steady state heat or mass transfer.

The most important consequence of

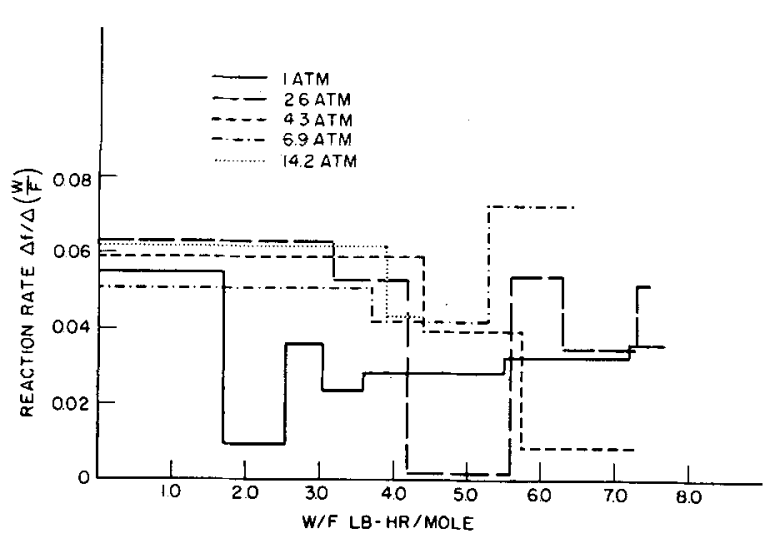

Fig. 2. Average rates for space-time increments.



Fig. 3. Original correlation for convection between a gas stream and a cylinder (5). 


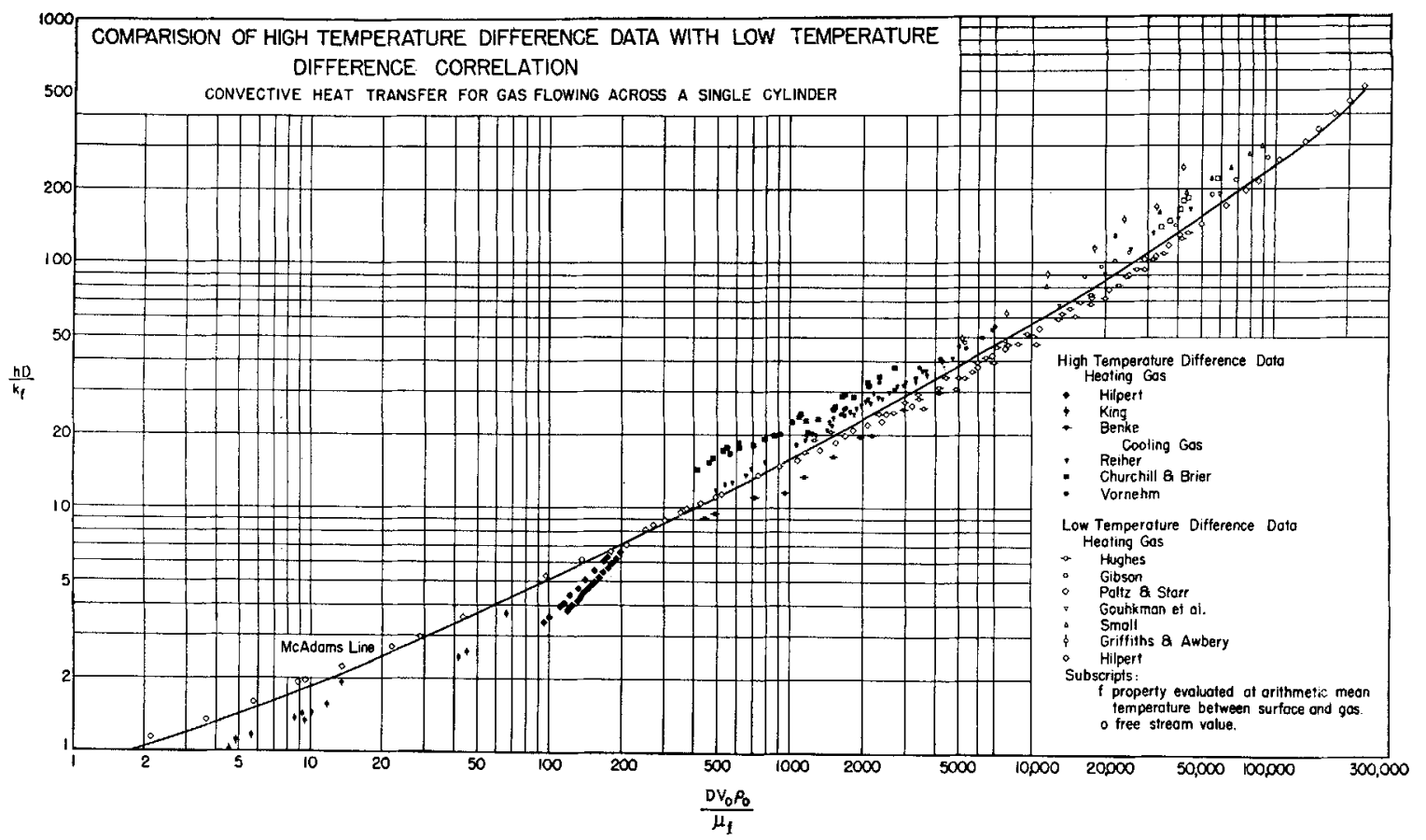

Fig. 4. Corrected correlation for convection between a gas stream and a cylinder ( 1 ).

Equations (3) and (4) is that in order to carry out the indicated integration, it is necessary to know the rate at which the process occurs as a function of the properties undergoing change. In the case of heat transfer this property is enthalpy or energy; in the case of mass transfer or chemical reaction it is composition.

\section{CORRELATION}

The primary purpose of correlation is to provide convenient and compact summaries of the relationship between the rate and the environment. This is the practical reason that scientists and engineers devote so much attention to the correlation of experimental facts and to the postulation of theories which provide models for the economical summary of the facts.

Generalization of rate data means that a set of measurements of a change can be associated with a description of the environment in which the change occurred. If a relationship between a rate of change and the environment can be ex- pressed with a reasonable economy of words or other symbols, a correlation results that simply an assumed cause-andeffect relationship between variables. The scope in relatively limited.

If the statement covers a somewhat limited field of knowledge, we say we have a theory, which is a picture invented to classify as far as possible but not necessarily completely the knowledge of a group of events and further to describe speculative results of observations yet to be made.

When a very large number of observa-

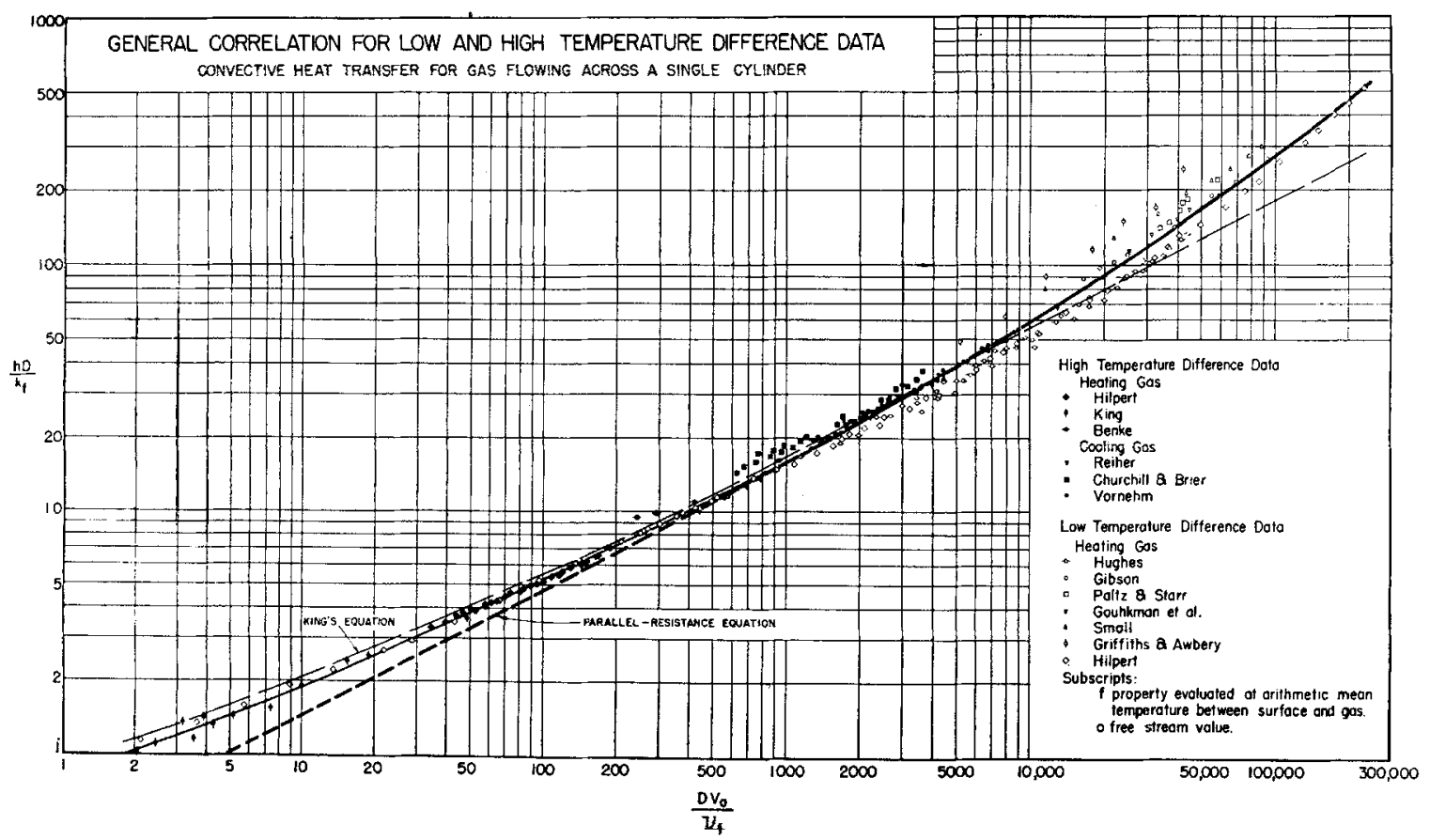

Fig. 5. Modified correlation for convection between a gas stream and a cylinder (1). 


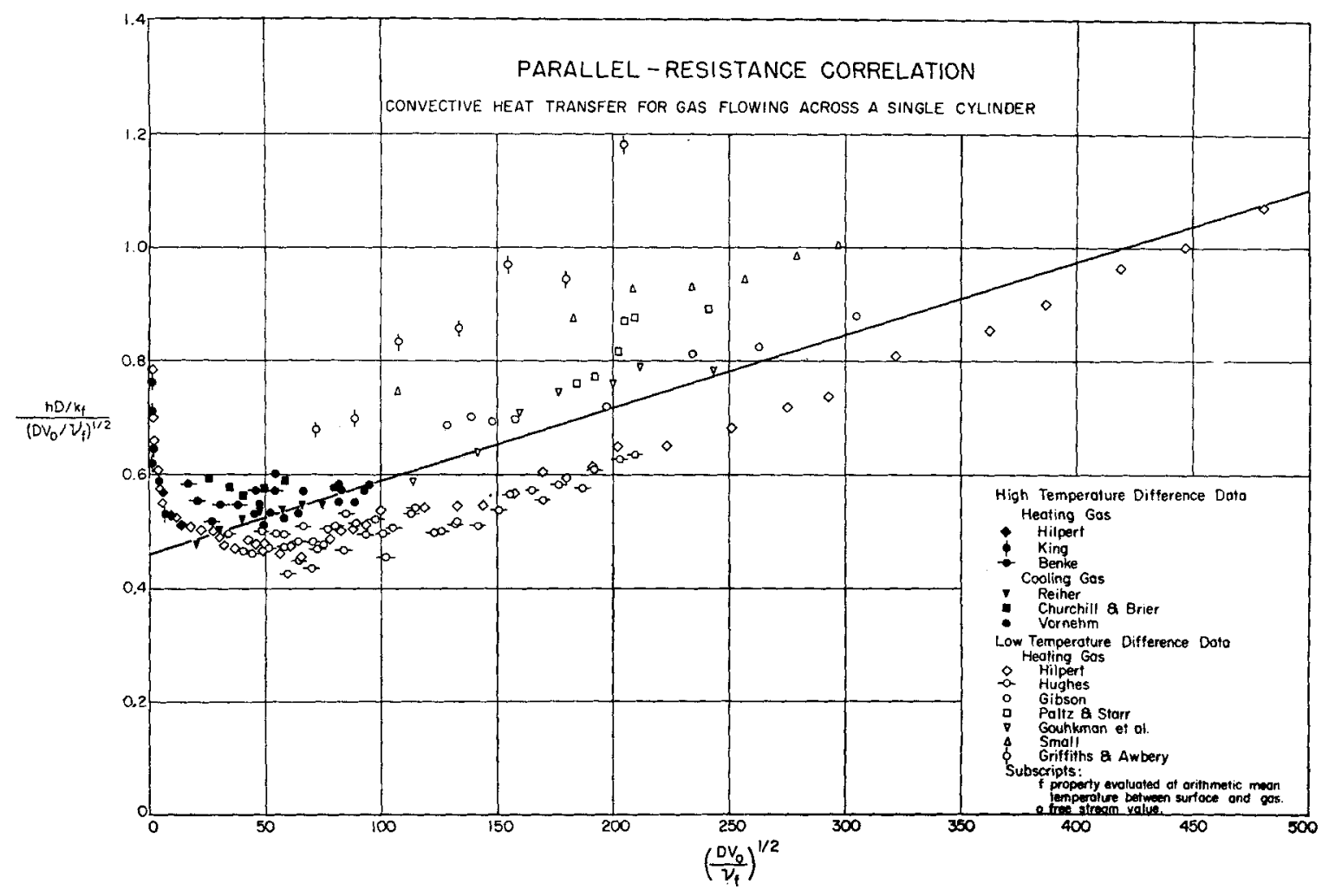

Fig. 6. Parallel-resistance correlation for convection between a gas stream and a cylinder (1).

tions show reproducible behavior, we may be able to make a general statement. If virtually no exception is found in our collective experience, and if the statement covers a relatively wide field of knowledge, it is then called law or principle. The statement of the first law of thermodynamics, "matter and energy cannot be created or destroyed," is an example. A law is useful because it describes in brief but complete form a very large body of knowledge. Obviously correlations lead to theories, which in turn lead to laws.

\section{ILLUSTRATIONS OF THE} CORRELATION PROBLEM

\section{Chemical Kinetics}

\section{Integral vs. differential representations}

Much of the previous discussion is illustrated by the data Figure 1 relating conversion to space velocity at a number of pressures for a catalytic decomposition. The reaction was of the type

$$
A \rightleftharpoons R+S
$$

with no identifiable side reactions.

The curves in Figure 1 are based upon a correlation of the form

$$
r=\frac{k\left[p_{A}-\frac{p_{R} p_{S}}{K}\right]}{1+K_{A} p_{A}+K_{R} p_{R}}
$$

and the summary of the paper from which the data are reproduced states, "It has been shown that a single site surface reaction is the controlling mechanism..."
The rate of reaction is defined by

$r=-\frac{d n}{d W}=-\frac{d\left(^{n} / F\right)}{d\left(^{w} / F\right)}=\frac{d f}{d\left(\left(^{w} / F\right)\right.}$

This definition indicates that the basic rate information consists of average rates.

$$
r_{\mathrm{avg}}=\frac{\Delta f}{d\left({ }^{w} / F^{\prime}\right)}
$$

These values of the average rate over finite steps of $w / F$ are shown in Figure 2 , the preparation of the data for which conversions at virtually the same value of $w / F$ were averaged.

The values of Figure 2 are the only known rates and constitute the raw material for correlation. They scatter widely and are typical of the data available from this type of experiment. The data, in fact, scatter so much that reasonably good correlations could be made on a zero-order basis, that is a straight horizontal line to represent all of the values, a somewhat better correlation on the basis of a simple first, second-orderreversible reaction, and perhaps a better correlation by Equation (6) as proposed by the experimenter. While the data can be represented adequately by Equation (6), there is a real question as to whether so complex a correlation is justified. It seems obvious that the reliability of the previously quoted conclusion as to mechanism is extremely dubious. The real point is that the presentation of the experimental results in the form of Figure 1 and Equation (6) without tabulation of the experimental data and without showing the rate values in Figure 2 may be extremely misleading not only to the scientist but especially to the perhaps less sophisticated practicing engineer.

There seems to be a strong psychological resistance to the differential treatment of data, particular on the part of those who have labored long, hard hours in the laboratory obtaining the data. The inconsistencies, errors, and uncertainties which the differential treatment brings to light constitute "bad news" to the experimenter, who, being human, wishes to view the results of his work in the most favorable light, as contrasted with the engineer, who in designing equipment wants to know the worst, since his design must allow for the uncertainties so that he may guarantee successful operation of the equipment. This conflict in viewpoint between the engineer and the laboratory is never ending and often difficult to resolve.

\section{Over Correlation}

The elucidation of the absolutereaction-rate theory (2) and the presentation by Hougen and Watson of this material in parctical form (B) has promoted a considerable volume of research and has led to the presentation of fairly extensive sets of chemical reaction-rate data for gas-solid systems. The correlations involve as many as five parametric constants. It was only recently pointed out (6) that many of these data can be expressed by simple two-constant kinetic relationships as well as by the multi- 
constant relationships reported by the investigators themselves. The improvement in the degree of correlation through the use of several additional constants is often negligible as compared with the intrinsic scatter of the data, and it is rather clear that little meaning can be attributed to the magnitude of the adsorption equilibrium constants, the frequency factor, apparent energy of activation, etc., obtained by fitting the data to such multiconstant equations.

\section{Heat Transfer}

\section{Improper Model}

Unrecognized shortcomings in the models used may also be responsible for poor correlation. For example, the usual definition of heat transfer coefficients for a double-pire exchanger involves the relation

$$
r=-\frac{d\left(u C_{p} t\right)}{d A}=h\left(t-t_{i}\right)
$$

This model, which ignores longitudinal transport due to eddy or back-mixing mechanisms, provides satisfactory correlations of $h$ in fluid dynamic regimes which approximate piston flow. When the same definition is applied to contacting equipment, such as packed towers and fluidized beds where extensive backmixing may occur, difficulty is encountered in obtaining general correlations of the transfer coefficients so defined. Present investigation of backmixing by means of response techniques may soon make it possible to obtain more satisfactory results when models which allow for back-mixing effects separately are used, as by

$k_{E} \frac{d^{2} t}{d z^{2}}-G C_{p} \frac{d t}{d z}=h\left(t-t_{i}\right)$

\section{Misinterpretation of Data}

The data for heat transfer to a gas flowing normal to a single cylinder provide an excellent example of the danger of the acceptance of correlations without examination of the raw data, as well as of the inhibiting effect of traditional models. The correlation for this system included in McAdams (5) is shown in Figure 3. It appears to be an excellent one. However examination of the raw data in the original references (mostly in German) revealed (1) that some of these points were transferred directly to Figure 3 without conversion of the physical properties from those used by the different authors to those in Figure 3. A corrected plot is shown in Figure 4. Unfortunately the ratio of the temperature of the surface to the temperature of the gas is now revealed to be a parameter. This error or its equivalent has been made in many other books on heat transfer. The parametric effect indicated in Figure 4 can be minimized by the use of $D V_{0} \rho_{f} / \mu_{f}$ as the abcissa instead of $D V_{0} \rho_{0} / \mu_{f}$ as shown in Figure 5.

\section{Inhibitions}

The curves through the data in Figures 3,4 , and 5 have definite curvature in $\log -\log$ coordinates, an indication that they cannot be represented by the favorite type of equation of chemical engineers:

$$
N u=A R e^{n}
$$

Nevertheless this equation has been recommended by almost all textbooks on heat transfer, with a whole series of values of $n$ and $A$ being given for different ranges of $R e$, such as those given in the legend of Figure 3. The representation of these same data by the equation

$$
N u=A \sqrt{R e}+B R e
$$

which can be rationalized on the basis of the summation of the contributions of heat transfer through a boundary layer on the forward portion of the cylinder and purely turbulent transfer through the wake on the rear portion,

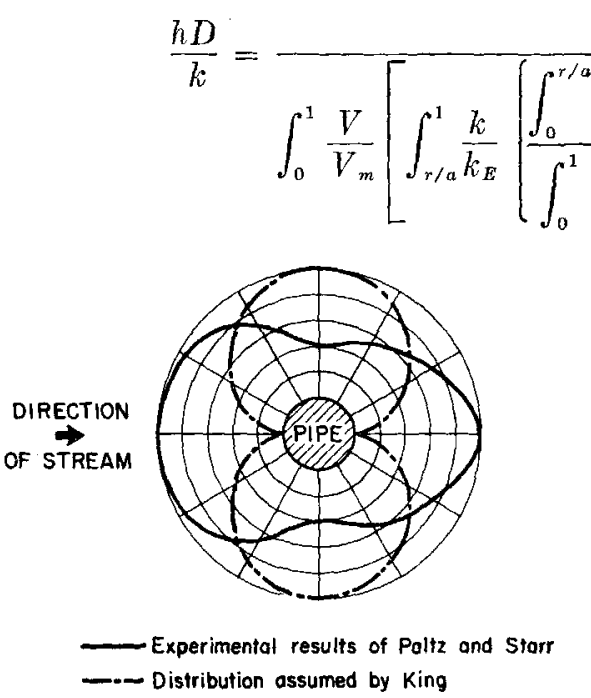

Fig. 7. Local rates of heat transfer between a gas stream and a cylinder $(5)$.

is illustrated in Figure 6. At first glance this is a poor correlation, but the coordinates here are linear instead of logarithmic and absolute deviations rather than percentage deviations are observable; the straight line in Figure 6 when replotted in logarithmic coordinates in Figure 5 appears to be a good representation except at low Re in logarithmic coordinates as shown in Figure 5 . Reexamination of the points in Figure 5 for low $R e$ indicates that they were all obtained at a sufficiently high $\Delta t$ so that natural convection may have led to higher transfer rates than forced convection, and the appearance of Figure 5 in the literature immediately produced correspondence from two investigators who had obtained data for low $\Delta t$ 's which agreed with the dotted line but which were not published because of failure to agree with McAdam's wellestablished curve.

\section{Integral Correlations as Tests of Dif- ferential Models}

Misleading conclusions are frequently drawn from integral correlations as opposed to differential correlations as suggested by the following examples. King (4) derived an expression for the local rate of convection to a cylinder by utilizing the theoretical flow pattern derived for potential (nonviscous) flow. The over-all rate obtained by integrating his expression is in reasonable agreement with the data in Figure 5, giving considerable credence to his expression for the local rates. However subsequently obtained experimental data for local rates reveal (Figure 7) that the agreement for the over-all coefficient was only fortuitous.

The differential energy balance resulting from an expression of heat transfer in fully developed turbulent flow in a pipe in terms of effective conductivities can be integrated formally to yield

2

$\left.\left.\frac{\frac{V}{V_{m}} \frac{\partial t}{\partial z} d\left(\frac{r}{a}\right)^{2}}{V_{m} \frac{\partial t}{\partial z} d\left(\frac{r}{a}\right)^{2}}\right] d\left(\frac{r}{a}\right)\right] d\left(\frac{r}{a}\right)^{2}$

This triple integration is a very effective smoothing operation, and as might be expected $h D / k$ proves to be quite insensitive to the velocity and eddy conductivity disturbances assumed in the various theoretical expressions which have been obtained by Von Karman, Martinelli, Lyon, Poppendick, etc., who integrated this equation by making various idealizations. $h D / k$ therefore is not a sensitive characterization of heat transfer rate data. This fact is also apparent from the observation that $h D / k$ approaches an asymptotic value in, say, ten pipe diameters while the temperature profile in the fluid is still developing thirty pipe diameters later.

\section{Momentum Transfer}

The differential momentum balances for a fluid, called the Navier-Stokes equations, have a great deal of gamevalue accuring from their complexity and virtual insolubility. However these equations and in particular the viscous stress terms which are derived on the basis of an assumed analogy between the shear and normal stress in a fluid and the stresses in a deformed plastic solid have not been tested experimentally. The system of momentum, energy, and material-balance equations if reducible to a single equation would contain highorder derivatives of the velocity. A critical test would therefore require 
instantaneous velocity-distribution data that would stand four or more differentiations, more precise data than have been obtained to date. The Navier-Stokes equations therefore consist of a more complex model for correlation than there are data to justify. Agreement between between experimental data and integral results obtained by analytical or numerical integration of the Navier-Stokes equations is subject to the same possibility of coincidence and the same problem of lack of sensitivity as illustrated above and cannot provide a critical test of the differential representation.

\section{Simulteneous Heat and Mass Transfer}

\section{The Wet-Bulb Temperature}

The problem of simultaneous heat and mass transfer through the same phase will be considered. Perhaps the simplest illustration of such a case is the socalled "wet-bulb temperature." If the wet-bulb process is described by a potential-difference model, the rate of heat transfer from the gas to the wick may be expressed as

$$
q=h A\left(t-t_{w}\right)
$$

and the rate of component transfer from the wick to the gas as

$$
r=k_{\mathrm{g}} A\left(p_{w}-p\right)
$$

At dynamic equilibrium the energy received by the wick from the gas must be equal to the latent heat of vaporization of the liquid at the wick temperature. Therefore

$$
h A\left[t-t_{w}\right]=k_{g} M A \lambda\left[p_{w}-p\right]
$$

which may be rearranged to give the so-called "wet-bulb depression" the difference between the dry-and wet-bulb temperatures:

$$
t-t_{w}=\frac{k_{0} M \lambda}{h}\left[p_{w}-p\right]
$$

Equation (17) has been reasonably successful as a correlation model for wet-bulb behavior. The values of $h / k_{\sigma}$ evaluated from wet-bulb measurements agree reasonably well with values calculated from the $j$-factor concept. While the use of wet-bulb thermometers is quite old, the amount of data available is surprisingly small and limited almost entirely to air systems. It is instructive to inquire somewhat more deeply into the correlation of such data, since they are closely related to other processes, particularly evaporation and drying. Because the data are limited and are well correlated by Equation (14), we cannot expect better correlation, but perhaps only a better insight into the mechanism of the transfer processes.

The logic by which Equation (17) was derived dodges a point which illustrates the basic difficulty of the potentialdifference model. The heat transfer coefficient in Equation (17) was defined in terms of the energy received by the wick from the gas. Obviously at equilibrium the heat transfer rate must equal the component transfer rate times the latent heat of vaporization of the liquid at the wet-bulb temperature, since the vaporized liquid leaves the interface at the wet-bulb temperature. However the vaporized liquid as it diffuses and mixes with the gas stream comes to the temperature of the gas streams, so that the total enthalpy change involved is $\lambda+$ $C_{\nu}\left(t-t_{w}\right)$.

The question therefore arises as to whether the heat transfer coefficient should be defined to include just the energy received by the wick or the total enthalpy. The reason for the question is of course that one would like to measure the heat transfer coefficient between air and a cylinder having the same geometry as the wick in the absence of component transfer and apply the coefficient to the performance of wet-bulb thermometers.

The only answer that can be given today is that heat transfer in the presence of component transfer is a different phenomenon from heat transfer in the absence of component transfer, and therefore there is no common meaning for the heat transfer coefficient. It is known that when the component transfer rate is small, the difference in the meaning of heat transfer coefficients is negligible compared with experimental error. However the potential-difference model remains uncomfortable.

These questions can be partially alleviated by the use of a potentialgradient model for the wet-bulb phenomena instead of the potentialdifference model. Consider a flat plane of infinite extent with countercurrent heat and component transfer by diffusion across a differential element $d y$ at a distance $y$ measured normal to the plane.

Under steady conditions there is no accumulation of enthalpy in the differential element due either to the temperature gradient or to the flow of enthalpy accompanying the flow of mass. Expressing this energy balance mathematically, one obtains

$$
k \frac{d^{2} t}{d y^{2}}=G C_{p} \frac{d t}{d y}
$$

At the interface, where $y=0, t=t$ and $k(d t / d y)_{y=0}=G \lambda$. Integrating equation (18) yields

$\ln \left[1+\frac{C_{p}}{\lambda}\left(t-t_{w}\right)\right]=\frac{G C_{p} y}{k}$

Equation (19) is somewhat disturbing in that as $y$ approaches $\infty, t$ also approaches $\infty$. This raises again the incompatibility of the potential-gradient and the potential-difference model. Obviously a heat transfer coefficient based upon the difference between a bulk value of $t$ and a surface temperature $t_{w}$ must be related to some finite value of $y$. The normal impulse is to say that the bulk value of $t$ is some value far removed from the surface, but this implies the selection of some arbitrary and large value of $y$ according to Equation (19). Perhaps it is not necessary to reconcile this difficulty in matching what may be unmatchable models. One evaluates $w$ in Equation (19) as a function of partial pressure so as to obtain a relation for the wet-bulb phenomena. If one follows a similar procedure in evaluating the mass transfer equation,

$$
G=\frac{\rho D_{f}}{y} \ln \frac{P-p}{P-p_{w}}
$$

Combining Equations (19) and (20) to eliminate $G$ gives the wet-bulb relationship

$t-t_{w} \frac{\lambda}{C_{p}}\left[\left(\frac{P-p}{P-p_{w}}\right)^{\rho D_{f} C_{p} / k}-1\right]$

Equation (21) correlates the available wet-bulb data as readily as Equation (17). However the comparison suggests a number of experiments designed to test the new model. A large factor is the ratio of $\lambda / C_{p}$ in Equation (21), which suggests an experiment to test whether the heat capacity of the vaporized liquid indicated by Equation (21) is important. Experiments in the hydrogen-carbon tetrachloride system with large values of $p$ approaching $P$ should therefore be interesting.

It is natural for the engineer to fall into a typical trap and to assume that because Equation (21) is a more "mathematical" model it is a better one. Only data can decide this. The use of Equation (21) for the data now available in the literature would represent overcorrelation, even though successful.

The basic difficulty with the potentialdifference models is further illustrated by consideration of simultaneous heat and mass transfer in a gas-liquid contactor, such as a dehumidifier. The mass balance around a differential section is

$$
d L=G d H
$$

and the mass transport relationship is

$$
G d H=k_{\imath} a M_{v}\left(p_{i}-p\right) d Z
$$

Similarly the enthalpy balance around the differential section is

$$
\begin{aligned}
G\{s d t+ & {\left[\lambda_{r}+C_{v}\left(t-t_{r}\right)\right.} \\
& \left.\left.-C_{l}\left(t_{l}-t_{r}\right)\right] d H\right\} \\
= & L C_{l} d t_{l}
\end{aligned}
$$

A flux balance around the interface of the differential section may be made by equating the rate of heat transfer through the gas and liquid phases to the interface and the enthalpy requirement for vaporizing the material at the interface, leading to

$$
\begin{aligned}
h_{\mathrm{v}} a\left(t-t_{i}\right) & +h_{l} a\left(t_{l}-t_{i}\right) \\
= & k_{g} a M_{\imath}\left(p_{i}-p\right) \lambda_{i}
\end{aligned}
$$


There is now a need for additional heat transfer relationships, and it is customary in the literature to write

$$
\begin{aligned}
-G s d t & =h_{v} a\left(t-t_{i}\right) d Z \\
L C_{l} d t & =h_{l} a\left(t-t_{i}\right) d Z
\end{aligned}
$$

Equations (23, 26, and 27) are substituted into (25),

$$
G\left[s d t+\lambda_{i} d H\right]=L C_{l} d t_{l}
$$

is obtained and comparison of Equation (28) with Equation (24) requires that

$$
\begin{aligned}
\lambda_{r}+C_{v}(t & \left.-t_{r}\right) \\
& -C_{l}\left(t_{l}-t_{r}\right)=\lambda_{i}
\end{aligned}
$$

Equation (29) leads to

$$
t-t_{i}=\frac{t-t_{l}}{1-C_{v} / C_{l}}
$$

since $0<C_{v} / C_{l}>1, t_{i}-t>t_{l}-t$; $t_{i}>t_{l}$ always. This cannot apply in a cooling tower with hot water and and cold dry gas.

Thus Equations (25) through (27) are basically incompatible. This results from the fact that while we can conceive of the right-hand side of Equations (26) and (27) as being the energy transferred to the interface, we cannot identify such heat in the enthalpy balance, since the enthalpy of the gas is a function both of temperature and composition and the entahlpy of the liquid is a function of its flow rate and its temperature. Arbitrarily segregating the so-called "sensible" heat part of the enthalpy change as indicated in Equations (26) and (27) is not operationally sound.

Thus if Equations (26) and (27) are used, we are in the impossible position of having to repeal the second law of thermodynamics as expressed by Equation (24) or to discard the concept of the flux balance as expressed by Equation (25). This constitutes a basic defect in the model, and while in the air-water system the defect is unimportant because the heat capacities are small as compared with the latent heat of vaporization, the defect may become very serious in other systems, such as hydrogen-carbon tetrachloride. Furthermore the defects may be serious when similar procedures are applied to the treatment of heat and mass transfer in catalytic reactors, where not only heat transfer but multicomponent mass transfer across the fluid phase are present. No satisfactory rationalization or model has been suggested to take care of this difficulty.

\section{CONCLUSIONS}

All the foregoing suggests that in chemical engineering we are still infants in treating adequately the complex problems encountered even in relatively simple processes. The profession suffers from a lack of data that will stand differentiation so as to give raw materials which are actual values of rates. Furthermore it suffers conceptually in that essentially all existing data and ideas are forced into two models, the potentialdifference and the potential-gradient models. The potential-difference models contain basic defects when applied in complex transport systems involving both heat and mass transfer; the potential-gradient models suffer because in their application to flow systems they lead to nonlinear, second-order, partialdifferential equations which are extremely difficult to handle mathematically and which place extreme requirements on the precision of the data which is necessary to evaluate the various terms. We tend to overcorrelate the data because we do not appreciate the intrinsic defects of the available rate information. This all suggests that the field of chemical engineering is still wide open for precise and careful expreimentation on relatively simple systems to provide the foundation for experimental evidence which by and large is still missing. One is reminded of the words of Mark Twain, who wrote in "Life on the Mississippi,"

In the space of one hundred and seventy-six years the lower Mississippi has shortened itself two hundred and forty-two miles. That is an average of a trifle over one mile and a third per year. Therefore, any calm person, who is not blind or idiotic, can see that in the Old Oolitic Silurian Period, just a million years ago next November, the lower Mississippi River was upward of one million, three hundred thousand miles long, and stuck out over the Gulf of Mexico like a fishing rod. And by the same token any person can see that seven hundred and forty-two years from now the lower Mississippi will be only a mile and threequarters long, and Cairo and New Orleans will have joined their streets together, and be plodding comfortably along under a single mayor and a mutual board of aldermen. There is something fascinating about science. One gets such wholesale returns of conjecture out of such a trifling investment of fact.

\section{NOTATION}

$A=$ area; aiso a constant

$a=$ radius of pipe; also surface area of packing per unit volume

$B=$ a constant

$C=$ heat capacity

$D=$ diameter

$D_{f}=$ diffusivity

$D_{0}=$ cylinder diameter

$d y=$ differential element

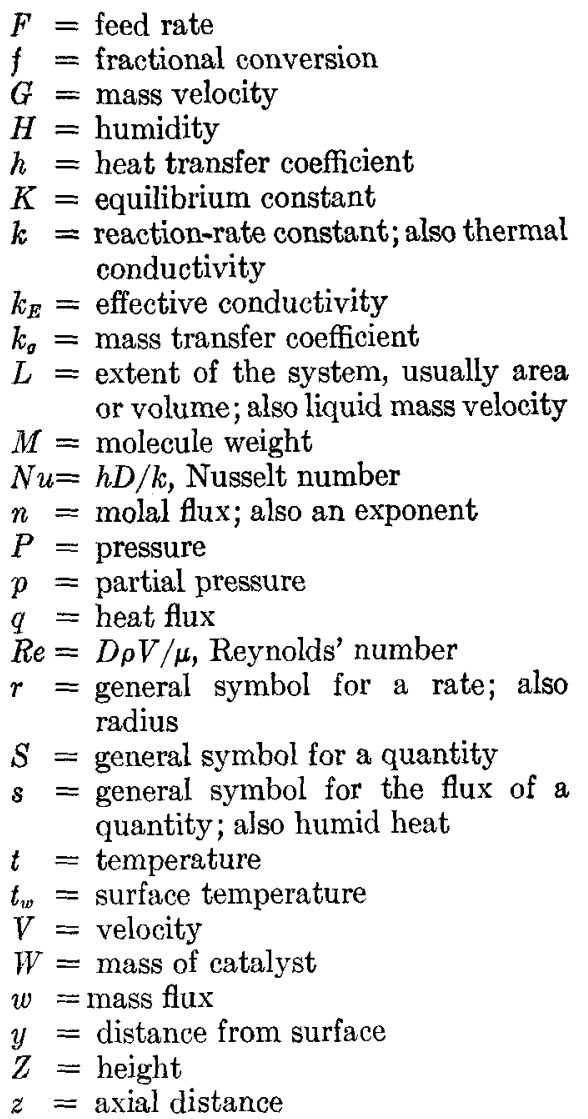

Greek Letters

$\lambda=$ latent heat of vaporization

$\mu=$ viscosity

$\rho=$ density

$\nu=$ kinematic viscosity

$\theta=$ time

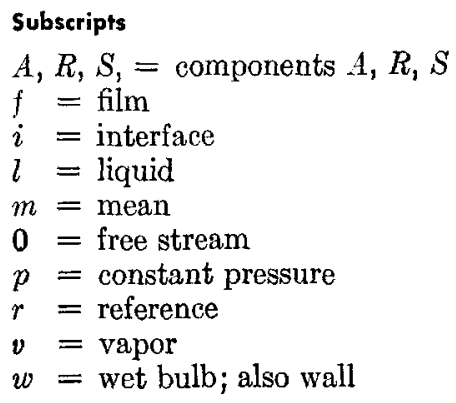

\section{LITERATURE CITED}

1. Douglas, W. J. M., and S. W. Churchill, Chem. Eng. Progr. Symp. Series No. $18,52,23$ (1956).

2. Glasstone, Samuel, K. J. Laidler, and Henry Eyring, "The Theory of Rate Processes," McGraw-Hill, New York (1941).

3. Hougen, O. A., and K. M. Watson, "Chemical Process Principles, Wiley, New York (1947).

4. King, L. V., Trans. Roy. Soc. (London), A214, 373 (1914).

5. McAdams, W. H., "Heat Transmission," 2 ed., McGraw-Hill, New York (1942).

6. Weller, Sol, A.I.Ch.E. Joumal, 2, 59 (1956).

Manuscript received August 14, 1958; revision received December 10, 1958; paper accepted December meeting. 